\title{
A Hybrid Peer Selection Scheme for Enhanced Network and Application Performances
}

\author{
Xu Zhang \\ Department of Electronic Engineering \\ University of Surrey \\ Guildford, Surrey GU2 7XH \\ Email:xu.zhang@surrey.ac.uk
}

\author{
Ning Wang \\ Department of Electronic Engineering \\ University of Surrey \\ Guildford, Surrey GU2 7XH \\ Email: n.wang@surrey.ac.uk
}

\author{
Michael Howarth \\ Department of Electronic Engineering \\ University of Surrey \\ Guildford, Surrey GU2 7XH \\ Email: m.howarth@surrey.ac.uk
}

\begin{abstract}
This paper presents a holistic peer selection scheme in multi-domain environments, aiming to mitigate Peer-to-Peer (P2P) traffic volumes over expensive inter-domain links as well as the maintenance of desirable P2P users' perceived service quality. The mechanism combines the traditional locality-aware peer selection with the consideration of ISP business relationship. By leveraging between the two peering strategies, the risk of possible congestion on critical inter-connected links can be effectively alleviated due to more concentrated P2P traffic over fewer interISP links under pure cooperative peering schemes. According to our analytical modelling, the proposed hybrid approach is able to achieve better performance for $\mathrm{P} 2 \mathrm{P}$ users, and can retain desirable network efficiency as of the cooperative peer selection strategy. Our modelling based analysis offers the incentives to perform peer selections in multi-domain environments wherein non-cooperative networks and cooperative networks coexist.
\end{abstract}

\section{INTRODUCTION}

Overlay applications, especially Peer-to-Peer (P2P) applications, have generated large volumes of traffic and account for a substantial proportion of the overall Internet traffic [17], leading to significantly costly inter-ISPs traffic for underlying networks and performance degradation for $\mathrm{P} 2 \mathrm{P}$ users at the same time. Numerous approaches have emerged in order to resolve the tussle between ISPs and P2P systems, such as ISPs' rate throttling and charging towards $\mathrm{P} 2 \mathrm{P}$ traffic, to which P2P systems response by encrypting their traffic or utilizing random ports to avoid being detected. The more recent proposition known as the locality-aware strategies suggests that optimised peer candidates should be selected in proximity to each client peer from the local underlying network, taking into account of the context information provided by overlyunderlay collaboration. These approaches have been claimed as efficient methods to reduce cross-ISP traffic while retaining desired P2P users' Quality of Experiences (QoE). [1]-[4] are representative examples of the localization-based mechanisms for peer selections, wherein a coordination entity located inside each ISP enables such peer selection ranking procedure by collecting relevant context information of the local underlying network topology for locality-aware peer selection operations, e.g. the ALTO framework proposed in IETF [5].

It is worth mentioning that, existing locality-aware approaches mainly focus on the environment of intra-ISP peer selections, while how to enable larger-scale collaborations across multiple autonomous ISP networks has not yet been comprehensively addressed in general. In particular, traditional locality-aware approaches, which refer to as the noncooperative strategy in this paper, mainly promote the selection of peers located within the same network, otherwise, potential peers in remote ASes (Autonomous Systems) with the shortest AS-hop are selected, but without distinguishing between these inter-domain paths regarding the diversity in business relationships among ISPs. A few works recently have been proposed suggesting that ISP business relationships should be taken into consideration in order to encompass the economic benefits of ISPs [6], [7]. These new approaches are referred as the cooperative strategy. While these approaches can effectively mitigate ISP costs among different inter-domain links, they are barely based on the hypothesis of an ideal all-cooperative environment. That is, adjacent autonomous ISP networks are all willing to cooperate with each other for the content object transferring. On the one hand, it is difficult to enable such ideal cooperative behaviours for all ISPs in practice, since some ISPs may not be willing to participate in such cooperation due to various reasons such as different operational objectives or simply privacy issues. On the other hand, even if there are incentives for such collaboration, potential risks can exist that $\mathrm{P} 2 \mathrm{P}$ traffic can be centralized over a small number of inter-ISP links under the pure localization promotion scenario, especially in the case of unlocalizable torrents, leading to possible congestion on critical inter-domain links and thus degradation of users' perceived service quality [19].

In this paper, we aim to address the aforementioned research issues by proposing an analytical model in order to provide accurate analysis on the following question: Is it always necessary to have an all collaborative peer selection strategy as current works have advocated? In particular, we analyse based on the multi-ISP network scenario the network performance and economic models of both ISPs and P2P systems, with systematic comparison between cooperative and non-cooperative network scenarios. By adopting stochastic methods, we characterize the different strategy options for the peer selection in a hybrid scenario as a number of stochastic states. An advanced hybrid peer selection scheme is then introduced for a more practical and realistic collaboration 
scenario. Our objective is to give incentives to perform peer selections in general multi-domain environments wherein noncooperative ISP networks and cooperative networks coexist, and on top of that, we present a comprehensive and accurate method for peer selections evaluations.

\section{RELATED WORK}

The schemes proposed in [1]-[4] are the representative paradigms to offer alternatives of pure locality-aware peer selection to improve ISPs efficiency and P2P systems performance by cooperation between the application layer and the network layer. Simulation-based analysis is mainly used to verify that cooperation between ISPs and P2P overlay systems is able to reduce cross-ISP traffic significantly while maintain desired download experiences for end users. This is typically achieved by introducing an entity coordinating between the P2P overlay and the underlying network, such as an oracle [1], [2] or by utilizing existing CDN [3] information. A solution is introduced in [4] to build an infrastructure-independent system to enable topology-aware BitTorrent Client, with an emphasis on downloading time and traffic reduction. The IETF Working Group is also dedicating their efforts to addressing the overlyunderlay interaction by proposing the ALTO (Application Layer Traffic Optimization) service [5], promoting a protocol that can be applied to enable enhanced communication between $\mathrm{P} 2 \mathrm{P}$ systems and network service providers. In this way, the traffic issues brought by $\mathrm{P} 2 \mathrm{P}$ applications can be alleviated while the $\mathrm{P} 2 \mathrm{P}$ users can benefit from such collaboration for better users' perceived service quality. However, all these solutions are confined to the benefits of a single ISP network without differentiation between individual domains regarding to various business requirements. While a few works proposed recently suggesting that peers in remote autonomous network systems should be ranked based on diversity ISP business requirements [6], [7],they are mainly on a basis of an assumption of a fully cooperative scenario, regardless of potential risks with respect to possible congestions over limited number of inter-domain links, and also the willingness to adopt such cooperation from individual ISPs. Consideration on the coexistence of non-cooperative and cooperative networks should be more realistic, but this has not yet received sufficient research attentions. Given the increasing complexity of the Internet topology, it becomes a more and more challenging task to optimise peer selections in the inter-domain scenario which coincide with the non-cooperative behaviours for performance enhancement on both the service side and the network side.

In this work, we use an analytical way to comprehensively investigate peer selection across multiple domains, focusing on a more realistic environment, with non-cooperative and cooperative policies coexisting in the scenario, rather than on the simple assumption of pure collaboration-based peering scenarios. Specifically, we take into account the preferences of not only local ISP's but also remote ISPs', with respect to business relationship among them. Additionally, we provide necessary attributes concerning both network performances and end user requirements. The analysis framework can be also used to systematically quantify the efficiency on both network and P2P user sides.

\section{SySTEM MODEL}

\section{A. Model Scenario}

A network scenario is illustrated in Fig. 1, depicting the practical environment that there are collaboration between overlay-underlay in some networks for the guidance of peer selection, such as $I S P_{A}$ and $I S P_{B}$, and also some network providers that are unwilling to cooperate, such as $I S P_{C}$. We assume that each ISP operates one single Autonomous System (AS) or domain, and hence we will use ISP network, domain and AS interchangeably in the rest of the paper.

Before presenting the peering procedure in such a mixed network scenario, we first consider how peer selection performs in a pure cooperative network scenario. The cooperative entity $(C)$ provides information regarding the context information of underlying network, which can be managed by an ISP itself or a trusted third party, such as the ALTO service [5]. For example in Fig. 1, $I S P_{A}$ connects with $I S P_{B}$ and $I S P_{C}$ directly, and a $\mathrm{P} 2 \mathrm{P}$ user $a$ in $I S P_{A}$ requests a content object. The $\mathrm{P} 2 \mathrm{P}$ tracker $(\mathrm{T})$ receives the queries and collects information about available peers spreading in the $\mathrm{P} 2 \mathrm{P}$ community, and then it communicates with the cooperative entity $(C)$ inside the $I S P_{A}$ for peer selection guidance by providing the information of the candidate peers, such as the IP addresses and users' access bandwidths capacities, etc. Upon receiving the information from $\mathrm{P} 2 \mathrm{P}$ systems, the entity $(C)$ performs the collaboration-based peering scheme by aggregating the information received from the underlying network $I S P_{A}$ of necessary context information of underlying network, such as traffic engineering policies, ISP business relationships and available bandwidths of certain inter-domain links, etc. At the same time, the entity in $I S P_{A}$ also needs to contact with the entity located in remote ISPs based on the cost requirements of individual inter-domain links. For instance, by communicating with $I S P_{B}, I S P_{A}$ can get additional information about remote peers, e.g., peer $b$ in $I S P_{B}$, in case the number of peers at local (inside $I S P_{A}$ ) are insufficient to satisfy user $a$ 's request. Upon receiving the necessary information from both users and underlying networks, the entity in $I S P_{A}$ performs the peer ranking procedure based on the preferences of both the application layer and the network layer. Then a list of ranked peers is returned back to the P2P tracker and finally retrieved back to the user $a$ to enable the content object transfer. For a trackerless network system, the peers communicate with the entity $(C)$ directly for potential peer connection guidance as shown in Fig. 1 with dashed ends lines. In this work, our results can be applicable to both situations.

Under the conventional collaboration promotion strategy based network scenario, peer selections mainly follow the procedure as mentioned above if all ISPs involved (e.g., $I S P_{A}$ and $\left.I S P_{B}\right)$ are willing to take part in the cooperation scheme. Nevertheless, there can be the case that some of the ISPs do not want to get involved into cooperation, such as stub ISPs with 


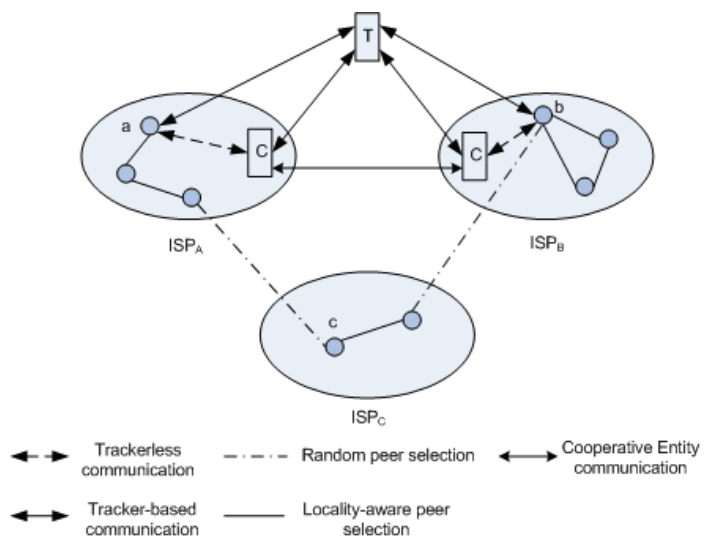

Fig. 1. An example of peer selections in the non-cooperative and cooperative coexistent network scenario.

no customer domains. Since in this case, they can only benefit from the relationship with their free peering domains, while the communication between stub ISPs and their respective provider ISPs for internet access for available peers in remote domains can incur costly transit traffic, which can lead to the economic benefits loss for stub ISPs. Due to large portions of P2P traffic flows over settle-free links [20], peering ISPs may have the incentives to tear up the free agreement between each other due to non-reciprocal benefits received regarding unbalanced traffic exchanged. Because of the risks, it is the stub ISP that suffers from the economic loss and thus is more willing to localize the P2P traffic within its own network rather than with remote ISPs if sufficient peers can be identified at local networks. Take $I S P_{C}$ in Fig. 1 as an example. Assume that $I S P_{C}$ adopts non-cooperative peer selections while $I S P_{A}$ and $I S P_{B}$ both adopt cooperative peering strategies. Available peers in the neighbouring networks from the view of user $c$, such as in $I S P_{A}$ and $I S P_{B}$, are the same to $I S P_{C}$ in terms of AS-hops and thus can be selected randomly without differentiations between them. In comparison, from the side of user $a$ or user $b, I S P_{A}$ and $I S P_{B}$ are able to distinguish between these external peers based on their respective business requirements, such that available peers in ISPs with higher business preferences can be considered with higher priorities in the ranking list. As such, while the economic benefits of ISPs can be achieved, potential risks can exist in the fully cooperative peering scheme. For example, the connections between $I S P_{A}$ and $I S P_{B}$ can be highly congested and thus constitute bottlenecks [19] if there is a preference business relationship between $I S P_{A}$ and $I S P_{B}$ for unlocalizable torrents requirements.

We propose a hybrid peer selection mechanism that can be used in a multi-domain scenario which is able to incorporate both non-cooperative and cooperative peering behaviours to address the above issues. This certainly requires necessary context information dissemination from the underlying ISP networks to the application-layer P2P.Given the availability of existing ISP-P2P collaboration paradigms such as ALTO [5], such context information can be certainly included, even though in this paper we will not specify how this will be practically realised. The hybrid peer selection procedure in the multi-domain scenario can be illustrated as follows. Users in $I S P_{A}$ generate queries, the outcome of which can be processed in mainly two ways under the hybrid peer selection mechanism.

(i) The queries can be considered to be processed by utilizing pure cooperation-based peering strategies, incorporating ISPs business relationships for external peer selection;

(ii) If critical links connecting external cooperative ISPs constitute bottlenecks by following the pure collaborative procedure, or the cooperative entities fail to operate their functionalities, non-cooperative of random peer selections without distinguishing between the same short inter-domain links can be applied alternatively, in order to achieve simple load balancing as well as to maintain the performance of P2P users'.

In particular, session (i) can be further extended to four processes on the basis of cooperative peer selections, with concerns of ISP business relationship [8]:

(a) Queries generated by users are first considered to be served inside the local ISP network. In case additional peers are required, then (b) they are served from its customer ISPS. If these are still insufficient, (c) queries are further served from peering ISPs. And finally (d) some peers are identified for serving the content which is located in provider ISPS or even further in the Internet which can be only reached via the provider ISP network. Such a peer selection strategy seems to be consistent with the current BGP routing policies driven by ISP's business relationships such that ISPs' economic cost and P2P users' perceived service experiences can be maintained.

Now we present an analytical modelling framework to analyse in a holistic way peer selection strategies across multiple domains based on the above concerns. Such a model not only takes into account of cooperative peering selections, but it also encompasses non-cooperative situations. The above prioritised peer selection strategy can be modelled in a stochastic method of several states, namely, state $(i)$, according to each target content object delivering environment. We refer the system state $x, x \in\{L, P, O\}$, to each state as customer ISPs $(L)$, peering ISPs $(P)$ or provider ISPs $(O)$ under the cooperative strategy, respectively, corresponding to each state $(i)^{1}$, except for the initial state (0). And states $\left\{L^{\prime}, P^{\prime}, O^{\prime}\right\}$ corresponds to the states under non-cooperative strategy, except for the state that network system is in a initial random peering decision making stage $\left(0^{\prime}\right)$. Assumptions and definitions are given first as bellow before we present our model.

Assumption 1: The mean time for peers participating in transferring desired objects in a state is independently and exponentially distributed, with mean $\lambda^{-1}$, where $\lambda$ is the transition rate to another state. Similarly the mean time for peers of successful downloading objects from a state is independent and exponentially distributed, with mean $\mu^{-1}$, where $\mu$ is the

\footnotetext{
${ }^{1}$ For simplicity we do not consider the scenario of selecting only local peers belonging to the same domain as the requesting peer.
} 
rate of a successful retrieval from the current state.

The dynamic of peer participation in a session can be modelled by a stochastic method, and the stochastic modelling is believed to be able to capture the fundamental characteristics and limitations of P2P streaming systems [9], [21], [22].

Definition 1: State $(i)$ denotes a case when a fraction of queries are served in state $(i)$ of the four cases illustrating all the possibilities of peer selection of P2P traffic, and we denote by $P_{i}(t)$ the probability that the process will be in state $(i)$ at time $t$, and the rate of transition from state $(i)$ to state $(j)$ is denoted as $R_{i j}$. Therefore, according to Markov process theories, we have the following differential equation

$$
\frac{d P_{i}(t)}{d t}=-\sum_{j \neq i} R_{i j} P_{i}(t)+\sum_{j \neq i} R_{j i} P_{j}(t)
$$

Definition 2: Heterogeneity of $\mathrm{P} 2 \mathrm{P}$ networks is defined here as diversity of uplink bandwidth capacities of users, and we denote by $\eta_{i}$ the uplink bandwidth of user $i$, and $p\left(\eta_{i}\right)$ the probability density function of $\eta_{i}$, thus we give the mean uplink bandwidth for users in state $x$ as

$$
E_{x}[\eta]=\sum_{i=1}^{p} p\left(\eta_{i}\right) \eta_{i}
$$

where $p$ is the number of categories of users' uplink bandwidths in state $x, x \in\left\{L, P, O, L^{\prime} P^{\prime} O^{\prime}\right\}$, and in state (0) and state $\left(0^{\prime}\right), p(\eta)=\sum_{j} \sigma_{j} \delta\left(\eta-\eta_{j}\right), \sigma_{j}$ is the percentage of one category of users' uplink bandwidth capacity. $j$ is the $j_{t h}$ classification of users' uplink bandwidth capacities. $\delta(\cdot)$ is the delta function.

According to measurement information used in [10], to model the heterogeneous P2P networks of unstructured P2P systems', one case of the users' uplink bandwidth distribution can be modelled as $p(\eta)=0.2 \delta(\eta-1)+0.45 \delta(\eta-10)+$ $0.3 \delta(\eta-100)+0.049 \delta(\eta-1000)+0.001 \delta(\eta-10000)$.

Definition 3: $\lambda_{x}$ denotes the transition rate to the next system state $x$, such as to the state of customer ISPs $(L)$, peering ISPs $(P)$ or provider ISPs $(O)$, and $\mu_{x}$ denotes the retrieval rate ratio that a query generated by a user results in a successful object retrieval from state $x$, respectively. Let $\gamma$ denote the fraction of online peers (e.g., peers are running at least one of P2P sessions.), and $N$ is the number of P2P users that share a common content object in the network, with average amount of requested data by a user in one request session at rate $q$, then $\lambda_{x}$ and $\mu_{x}$ can be respectively denoted as

$$
\begin{gathered}
\mu_{x}=\frac{n_{x} E_{x}[\eta]}{N q} \gamma \\
\text { where } x \in\left\{L, P, O, L^{\prime}, P^{\prime}, O^{\prime}\right\} . \\
\lambda_{x}=\frac{q}{n_{x-1} E_{x-1}[\eta] \gamma}= \begin{cases}1 & n_{x-1}=0 \\
\lambda_{x} & n_{x-1}>0 . \\
\text { where } x \in\left\{L, P, O, L^{\prime}, P^{\prime}, O^{\prime}\right\} .\end{cases}
\end{gathered}
$$

wherein $n_{x}$ is the number of peers participating transferring objects in state $x$. State $x-1$ means the previous state the

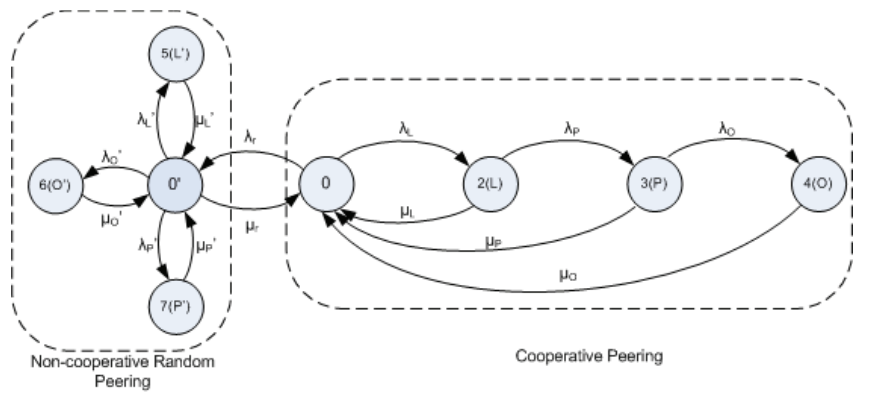

Fig. 2. Continuous Time Markov Chain (CTMC) of the hybrid peer selection procedure in a multi-domain scenario.

system is in before transit to current state $x$, and state $L-1$ (or $L^{\prime}-1$ ) refers to the local network. $n E[\eta] \gamma$ represents the capacities of available peers in the local network.

Note in Eq. (4) that $\lambda_{x} \propto \frac{q}{n_{x-1}}$, which means that the more the number of available peers $n_{x-1}$ in state $x-1$, the smaller the probability of peer selections in the next state $x$, in the premise that $n_{x-1} \neq 0$, while in Equation (3) $\mu_{x} \propto n_{x}$, which means that the more the number of peers in state $x$, the higher the retrieval rate from that state is. In special cases that the number of peers in a system state equals to 0 (e.g., a stub ISP with no customer ISPs subscribing to), leading to $n_{x-1}=0$ in Eq. (4), then transition rate follows $\lambda_{x}=1$. Take Fig 1 for example, $I S P_{A}$ is where queries are generated, and if there are no available peers in its customer ISPs (or $I S P_{A}$ is a stub ISP), the potential peers in its peering ISPs will be considered with transition rate from its customer ISPs to its peering ISPs equal to 1 . This relationship between $\lambda_{x}$ (or $\mu_{x}$ ) and the number of peers is reasonable, since for peer selection procedure related to business relationships of ISPs, local choices are always preferable, and the more the peers participating in traversing desired objects, the faster the downloading rate back to the users, in the premise that $n_{x-1} \neq 0$. Also in the case that the network is a Tier-1 network without further provider ISPs, then Eq. (3) equals to 0 for $x \in\left\{O, O^{\prime}\right\}$. So our proposal in this paper is applicable to general ISPs, including stub ISPs without customers and ISPs without providers.

\section{B. Markov Model}

We present in Fig. 2 the dynamics of hybrid peer selection procedure from $I S P_{A}$ 's perspective with concerns of cooperative and non-cooperative peering scenarios. We assume that the initial state is in state $(0)$, in which case local peers belonging to the same domain are first selected. Since our study only focuses on the peer selection strategies associated with remote ISP networks, such an initial state involving only local peers is regarded as the starting point of the modelling. If critical inter-ISP links are highly congested under the cooperative peering strategy, the network will transit towards the non-cooperative peering process (state $\left(0^{\prime}\right)$ ) at transition rate $\lambda_{r}$ alternatively. Otherwise, the system will follow the collaboration-based peering scheme incorporating ISP business relationships following the order of inside ISP 
(0)-local ISPs $(2(L))$-peering ISPs $(3(P))$-provider ISPs $(4(O))$ as described previously. Thus the $\mathrm{P} 2 \mathrm{P}$ peer selection procedure can be modelled by a Markov chain with several states; the system is in state $x$ if the queries are not able to be resolved in the previous state $x-1$. For example, state (0) expresses that the queries generated by users are supposed to be first served within the $I S P_{A}$ they subscribe to. If the peers in this community cannot provide sufficient connectivity, a fraction of the quires will be transferred to state $(1(L))$, the customer ISPs, at transition rate $\lambda_{L}$. In state $(1(L))$ content objects can be downloaded and retrieved back to state $(0)$ at $\mu_{L}$ and the request and response process for users is finished if sufficient peers can be found. However, if critical interISP links connecting $I S P_{A}$ and other cooperative networks constitute bottlenecks, or the cooperative entity managed by the network encounters failure operations, non-cooperative peer selections (states $\left.\left(0^{\prime}\right),\left(L^{\prime}\right),\left(P^{\prime}\right),\left(O^{\prime}\right)\right)$ will be then considered alternatively to alleviate the inefficiency of pure collaborative peering scheme, at transition rate $\lambda_{r}$, in order to maintain users' perceived service quality and the network performance as well.

Then we can obtain a set of differential equations corresponding to the model according to Equation 1 as

$$
\left\{\begin{aligned}
\frac{d P_{0}(t)}{d t} & =-\left(\lambda_{L}+\lambda_{r}\right) P_{0}(t)+\mu_{r} P_{1}(t)+\mu_{L} P_{2}(t) \\
& +\mu_{P} P_{3}(t)+\mu_{O} P_{4}(t) \\
\frac{d P_{1}(t)}{d t} & =\lambda_{r} P_{0}(t)+\mu_{L^{\prime}} P_{5}(t)+\mu_{O^{\prime}} P_{6}(t) \\
& +\mu_{P^{\prime}} P_{7}(t)-\left(\mu_{r}+\lambda_{L^{\prime}}+\lambda_{O^{\prime}}+\lambda_{P^{\prime}}\right) P_{1}(t) \\
\frac{d P_{2}(t)}{d t} & =\lambda_{L} P_{0}(t)-\left(\lambda_{P}+\mu_{L}\right) P_{2}(t) \\
\frac{d P_{3}(t)}{d t} & =\lambda_{P} P_{2}(t)-\left(\lambda_{o}+\mu_{P}\right) P_{3}(t) \\
\frac{d P_{4}(t)}{d t} & =\lambda_{O} P_{3}(t)-\mu_{O} P_{4}(t) \\
\frac{d P_{5}(t)}{d t} & =\lambda_{L^{\prime}} P_{1}(t)-\mu_{L^{\prime}} P_{5}(t) \\
\frac{d P_{6}(t)}{d t} & =\lambda_{O^{\prime}} P_{1}(t)-\mu_{O^{\prime}} P_{6}(t) \\
\frac{d P_{7}(t)}{d t} & =\lambda_{P^{\prime}} P_{1}(t)-\mu_{P^{\prime}} P_{7}(t)
\end{aligned}\right.
$$

Solving the set of Eq. (5) with the initial conditions $P_{0}(0)=1$ and $P_{i}(0)=0, i \in\{1, \ldots, 7\}$, along with the boundary condition $\sum_{i=0}^{7} P_{i}(t)=1$ yields the probability that the process will be in state $(i)$ at time $t, P_{i}(t), i \in\{0,1, \ldots, 7\}$.

The values of $\lambda_{x}$ and $\mu_{x}$ can be determined according to Eq. (3) and (4). For the values of $\lambda_{r}$ and $\mu_{r}$ related to non-cooperative process in Fig. 2(b), they can be denoted as $\lambda_{r}=\frac{k^{\prime}}{k I_{(\theta \neq 0)}}=\left\{\begin{array}{cc}\frac{k^{\prime}}{k} & \theta \neq 0 ; \\ 1 & \theta=0 .\end{array}, \mu_{r}=\frac{n_{r} E_{r}[\eta]}{N q}\right.$, respectively, wherein $\theta$ indicates the probability of cooperative entity perform its function normally. $I_{(\theta \neq 0)}$ is the indicator function, and equals to 1 if the conditions are met and 0 if not, and $\frac{k^{\prime}}{k}$ represents the congestion ratio constituted by $\mathrm{P} 2 \mathrm{P}$ traffic on inter-ISP links, wherein $k^{\prime}$ is the number of inter-ISP links congested under pure cooperation-based peering strategy and $k$ is the number of inter-ISP links utilized by P2P traffic. $n_{r} E_{r}[\eta] \gamma$ illustrates the capacities of potential peers selected based on non-cooperative peer selections.

Note that if we remove the transition rates $\left(\lambda_{r}, \mu_{r}\right)$ from the model in Fig 2(b), then the figure becomes two separate mod- els, which is the non-cooperative peering model and the model describing the pure cooperation-based peer selection procedure with concerns of ISPs business relationships, respectively.

\section{Attribute Models in Peer Selections}

We give a series of metrics in this section to evaluate the hybrid peer selection strategy, with respect to performance attributes from both networks' and P2P users' perspectives, along with ISPs economic cost taking into consideration.

1) ISP Efficiency: ISP efficiency here refers to the ISPs' capability to control P2P traffic in an optimised manner. As previously mentioned, we mainly consider the key objective of reducing $P 2 P$ content traffic across inter-domain transit links while maximising business revenues. Now we first consider ISP efficiency that only concerns P2P traffic reduction, leaving the business objective of maximising revenue to section 4.3 ISP Economic Benefits. In this case, higher the ISP efficiency indicates lower the $\mathrm{P} 2 \mathrm{P}$ traffic volume over transit links, such that the network resources are better utilized. By adopting localization strategies ISPs are able to reduce cost over transit links connecting to provider domains. Thus the main objective of localization is to maximize the probabilities for states $\left(0^{\prime}\right)$, $\left(L^{\prime}\right),\left(P^{\prime}\right),(0),(2),(3)$, which can be used to represent ISP efficiency here as the following equation.

$$
e_{I S P}=1-\left[P_{O}(t)+P_{O^{\prime}}(t)\right]
$$

In this case, the higher the probabilities of process in states $\left\{\left(0^{\prime}\right),\left(L^{\prime}\right),\left(P^{\prime}\right),(0),(2),(3)\right\}$ are, the higher the ISP efficiency will be, benefiting ISPs in terms of efficient network resources utilizations.

2) P2P User Efficiency: P2P user efficiency here indicates the experiences for individual users to successfully download the desired content object. It is easy to derive that the bigger the aggregated retrieval rate of each state in Fig 2, the higher the successful downloading probability, indicating better downloading experiences for users. Thus we use the aggregate value of $\mu_{x}$ to denote the P2P user efficiency according to Equation (3), which can be expressed as

$$
\mu=\frac{n E[\eta]}{N q} \gamma+\sum_{x} \mu_{x}=\frac{n E[\eta]}{N q} \gamma+\sum_{x} \frac{n_{x} E_{x}[\eta]}{N q} \gamma
$$

where $x \in\left\{L, P, O, L^{\prime}, P^{\prime}, O^{\prime}\right\}$.

It is generally observed that most network bottlenecks in the Internet are assumed to be either in the access network or on the inter-domain links between ISPs [11]. Since a significant proportion of the overall Internet traffic is generated by P2P applications [12], the majority of which traverses multiple inter-ISP links rather than in intra-ISP links [13], there is a necessity to confine the $\mathrm{P} 2 \mathrm{P}$ traffic within the bandwidth capacities of inter-ISP links. We thus mainly consider the bottlenecks between inter-ISP links in this paper. In this case Equation (7) becomes

$$
\mu=\frac{n E[\eta] \gamma+k_{c} \overline{B_{u}}+m \overline{B_{p}}+k \overline{B_{d}}}{N q}
$$


where $k_{c}$ is the number of inter-domain links connecting customer ISPs to provider ISP, $m$ is the number of peering links connecting peering ISPs, $k$ is the number of transit links, $\overline{B_{y}}$ is the average bandwidth of links connecting two ISPs, $y \in\{u, d, p\}$, which describes customer-provider upstream connection for $u$, provider-customer downstream connection for $d$, and peering connection for $p$, respectively.

We have the following equation, referring to user utility, to determine the lower bound of $\mu$, which indicates the minimal economic benefits of individual users towards the service perceived form the relevant network.

$$
U_{s}=\log \left(\alpha_{s} \mu+1\right)-c
$$

where $\alpha_{s}$ is a shape parameter related to a particular user. $c$ is a fixed price that users subscribing to the ISP have to pay. Notice that $U_{s}$ is a strictly concave function in $\mathrm{i}$ and as noted in [14], a concave function is commonly used for describing elastic traffic, which is the dominant traffic in the Internet, and is also common used for performing distributed admission control [15].

The log function is chosen to model diminishing returns as $\mu$ increases, so $\mu=0$ yield no benefits to the user, thus a minimum can be derived as $U_{s} \geq 0 \Rightarrow \mu \geq\left(e^{c}-1\right) / \alpha_{s} \Rightarrow$ $\mu_{\text {min }}=\left(e^{c}-1\right) / \alpha_{s}$.

A lower bound of the number of inter-ISP links is given below to satisfy P2P users' perceived service quality.

Theorem 1: Given the states of available peers at local in terms of $n E[\eta] \gamma$ and the average value of inter-ISP bandwidths occupied by $\mathrm{P} 2 \mathrm{P}$ traffic, $\bar{B}$, a minimum number of inter-ISP links exists to maintain the benefits of $\mathrm{P} 2 \mathrm{P}$ users (in terms of service quality, $\mu_{\min }$ ) subscribing to an ISP, which can be given as

$$
k_{\min }=\frac{\mu_{\min } N q-n E[\eta] \gamma}{\bar{B}}
$$

Proof: According to Equation (9), a user can be benefited from the network subscribed only if $U_{s} \geq 0$. Then we can deduce that $\mu_{\min }=\left(e^{c}-1\right) / \alpha_{s}$ for $U_{s} \geq 0 \Rightarrow \mu \geq$ $\left(e^{c}-1\right) / \alpha_{s}$. Replace $\mu$ with $\mu_{\text {min }}$ in Equation (8) and we can get $\mu_{\min }=\frac{n E[\eta] \gamma+k_{c} \overline{B_{u}}+m \overline{B_{p}}+k \overline{B_{d}}}{N q}$. Let the average inter-ISP bandwidth be $\bar{B}$, then the equation of $\mu_{\min }$ is transformed as $\mu_{\text {min }}=\frac{n E[\eta] \gamma+k \bar{B}}{N q}$ for $k$ inter-ISP links. Given the states of peers inside the network, then $k_{\min }=\frac{\mu_{\min } N q-n E[\eta] \gamma}{\bar{B}}$ can be obtained accordingly.

This is an important result since potential risks can be avoided with respect to congestion on inter-domain links. For instance, in the case of large $\mathrm{P} 2 \mathrm{P}$ traffic volumes in a native cooperative peering scenario, which can be general due to the explosive growth of P2P traffic, the possibility of congestion on critical inter-ISP links can be high due to centralized P2P traffic over fewer inter-ISP links. Instead, more interISP links can be adopted to avoid bottlenecks under hybrid peering scenarios for random peer selections in inter-domain are deployed if critical inter-ISP links are highly congested.
On the one hand, the hybrid peering strategy can mitigate the congestion probability over critical inter-ISP links and thus guarantee a desired network performance and fairness among other overlay applications. On the other hand, the hybrid scheme can perform its functionality well even in the case that cooperative entities fail to operate due to unexpected events, e.g., marital attacks or critical components failure, such that a desirable performance for P2P users can be maintained.

3) ISP Economic Benefits: We next investigate the revenue generated by an ISP for carrying P2P traffic. In general, an ISP receives revenue from its subscribers (including customer domains and end users) and pay for the connection to its provider ISP. The economic cost of an ISP consists of mainly two parts: 1) peering cost, a fixed cost of providing bandwidth from its peering ISP (e.g., for a peering port fee), which is ignored in our work compared to transit cost; and 2) transit cost, $C_{j}^{d}$, which is a transit cost for each unit of bandwidth to the provider ISP1, proportional to the mean allocated bandwidth $\overline{B_{d}}$. For simplicity we assume that there is an identical charge for both outbound and inbound traffic between a customer ISP and a provider ISP. Therefore, an $I S P_{i}$ 's profit can be expressed by

$$
\begin{aligned}
& U_{I S P_{i}}=\left(\sum_{s=1}^{n} c_{i} I_{\left(U_{s} \geq 0\right)}+\overline{B_{d}} \sum_{z=1}^{k_{c}} C_{z}^{d} I_{\left(R_{c_{-} z} \neq 0\right)}\right) \\
& -\overline{B_{d}} \sum_{j=1}^{k} C_{j}^{d} I_{\left(R_{O_{-} j} \neq 0\right)}
\end{aligned}
$$

where $I_{(\cdot)}$ is the indicator function, and equals to 1 if the conditions are met and 0 if not. Parameter $n$ is the number of users subscribing to $I S P_{i} . R_{c_{-} z}$ and $R_{O_{-} i}$ represent the traffic over links of from customer ISPs to the current ISP and via transit links, respectively, $R_{O_{i} i}=0$ expresses that there is no traffic over transit links connecting its provider domains, and similarly $R_{c_{-} z}=0$ means there is no traffic over the links connecting its customer domains (if any). $\sum_{s=1}^{n} c_{i} I_{\left(U_{s} \geq 0\right)}$ illustrates the cost paid by users subscribing to $I S P_{i}$. Term $\sum_{z=1}^{k_{c}} C_{z}^{d} I_{\left(R_{c_{z}} \neq 0\right)}$ refers to the revenues $I S P_{i}$ generates from its customer ISPs if there are traffic flows over the customerprovider links. $\sum_{j=1}^{k} C_{j}^{d} I_{\left(R_{O_{-}} \neq 0\right)}$ indicates that the ISP needs to pay transit fees if there are P2P flows exchanged between itself and its $k$ multi-homed transit provider ISPs.

It is difficult to guarantee the desired economic benefits for an ISP in a hybrid peering scenario as that in a native cooperative peering scenario. The reason is that under hybrid peer selection scenario, random peering scheme in interdomain is considered, which can lead to the values of the term $\sum_{z=1}^{k_{c}} C_{z}^{d} I_{\left(R_{c_{z}} \neq 0\right)}$ and the summation $\sum_{i=1}^{k} C_{i}^{d} I_{\left(R_{O_{-}} \neq 0\right)}$ increase at the same time in Equation (11).

However, a condition can be given as below to illustrate the relationship of the number of $k$ and $k_{c}$ to explain how to maintain the benefits of an ISP under the hybrid peering scheme.

Corollary 1: Given the number of available peers inside a network and bandwidths of inter-ISP links, a relationship between the number of transit links (provider-to-customer and 
customer-to-provider) exits to maintain the benefits for ISPs. The relationship can be expressed as

$$
k_{d}-k_{c} \leq \frac{n c}{\overline{B_{d}} C^{d}}
$$

For stub ISPs, without no customer domains subscribe to, that is, $k_{c}=0$, then a range for the number of inter-ISP links can be derived to maintain the utility for both ISPs and users, which can be given as

$$
k \in\left[\frac{\sigma_{\min } N q-n E[\eta] \gamma}{\bar{B}}, \frac{n c}{\overline{B_{d}} C^{d}}\right]
$$

Proof: $U_{I S P_{i}}>0$ gives the condition for an ISP to be minimally benefited according to Equation (11). Then given number of available peers inside a network, $U_{I S P_{i}}>0 \Rightarrow$ $k_{d}-k_{c} \leq \frac{n c}{\overline{B_{d} C d}}$. If the ISP is a stub ISP that has no customer ISPs subscribing to, $k_{c}=0$, yielding the upper bound value of inter-ISP links' number $k=k_{d} \leq \frac{n c}{\overline{B_{d}} C^{d}}$. According to Theorem 1, the lower bound for inter-ISP links can be obtained as $k \geq \frac{\sigma_{\min } N q-n E[\eta] \gamma}{\bar{B}}$. Thus the range for inter-ISP links can be derived to maintain ISPs' profit and satisfy user's quality of service at the same time.

Equation (12) and (13) express the condition that interdomain links need to be confined in a specific range in order to achieve desirable performance for users and to maintain economic profit for network systems at the same time.

\section{NumERICAL ANALYSis}

We specify the setting of experiment parameters below, and unless specified the values will keep fixed throughout the experiments. According to [16], the total number of concurrent users over the Internet sharing a popular content object can be assumed to be at the magnitude of 10,000.

- $N=10^{4}$. The number of P2P users in the considered model which share a common content object.

- The average value of bandwidth between ISPs is $\bar{B}=10,000$ Mbps.

- The value of $\gamma$, according to [1], the on-line fraction of peers can be in the range of $80-75 \%$, so we set $\gamma=0.8$ here, assuming that most of peers are on-line, and willing to share contents with each other.

- We assume the average value of users' access capacities can be calculated as $E_{x}[\eta]=1$ Mbps. Since the upload bandwidth capacity of many users is much lower than the download bandwidth capacity based on ADSL environment, it is reasonable to assume the average user's access capacity is around $1 \mathrm{Mbps}$, compared to $10 \mathrm{Mbps}$ of download capacity.

- We assume a unified cost for users subscribing to ISP is $\bar{c}=1$, the mean shape parameter is $\overline{\alpha_{s}}=5$, thus the minimum rate efficiency can be obtained according to Equation (10) as $\mu_{m i n \_i}=\left(e^{\bar{c}}-1\right) / \alpha_{s}=0.3$, which means that an amount of users are minimally satisfied if one third of their queries can be retrieved successfully under the above assumption. The value of this parameter can be further tuned according to the sensitivity of the required object by users, such as on-line video sharing, wherein the value of $\mu_{\text {min }}$ can be set higher than the native file sharing (e.g., file downloading rather than real time video streaming, etc.) to meet users' experiences.

\section{A. Network Efficiency and Economic Benefits Evaluation}

We compare in Fig. 3 the ISP efficiency $\left(e_{I S P}\right.$, defined in Eq. (6)) under different peering strategies for the first 20 time intervals (time intervals are used here to show the dynamicity of the P2P system based on CTMC, and $t=0$ is the time point at which the CTMC starts, and is in its initial state), respectively, with $\mu_{L}=\mu_{L^{\prime}}=0.7, \mu_{P}=\mu_{P^{\prime}}=0.8$, $\mu_{O}=\mu_{O^{\prime}}=0.9, \mu_{r}=0.5$ and $\lambda_{L}=\lambda_{L^{\prime}}=0.9, \lambda_{P}=\lambda_{P^{\prime}}=$ $0.8, \lambda_{O}=\lambda_{O^{\prime}}=0.7, \lambda_{r}=0.01$ fixed. The value of $\mu_{x}$ corresponds to the fact that there are more peers holding the desired content in peering or the Internet than those at local [18], while the value of $\lambda_{x}$ set as above is to consistent with our peering promotion that local peers are considered with high priority. As shown in the figure, network efficiency is declining before reaching a steady state with time elapses. This observation verifies the statement in [16] that the distribution of peers is highly skewed. In other words, only a small number of content objects accounts for the majority of downloads which are not located in the same ISP for most peers [18]. As such, the results are able to reflect the real $\mathrm{P} 2 \mathrm{P}$ network situation. Note that the hybrid peering scheme performs similarly in terms of network efficiency to the cooperative strategy by mitigating a certain amount of the transit traffic as compared to the noncooperative strategy as shown in the figure. Fig. 4 shows that the network efficiency under the hybrid peering strategy can be slightly decreased with the value of $\lambda_{r}$ increasing, since non-cooperative is adopted with higher probability, without distinguishing between different ISPs.

Inter-ISP traffic mitigation ratio is depicted in Fig. 5. The hybrid peer selection scheme can maintain similar performance as the native cooperative peering scheme as shown in the figure, while outperforms the non-cooperative scheme significantly since randomized peering scheme is adopted to select neighbouring ISPs with the same short AS-hops. The results indicate that the hybrid peering strategy preserve the promising effectiveness as the native cooperation-based scheme to alleviate the cross-ISP traffic. On the other hand, the slight decrement of the value of Inter-ISP traffic mitigation ratio under hybrid peering scheme indicates that the $\mathrm{P} 2 \mathrm{P}$ traffic volumes are not strictly confined within the local network but rather can have bigger chance to rely on a little more inter-domain links compared to the cooperative strategy, thus relieving possible burdens on critical costly inter-connections between ISPs that may cause congestions, which is consistent with our previous analysis.

Since the main purpose for such hybrid peer selections proposition is to alleviate $\mathrm{P} 2 \mathrm{P}$ traffic intensity over critical inter-ISP links, the probabilities $\mathrm{P} 2 \mathrm{P}$ traffic traversing cross via transit links could be increased, as shown in Fig. 3 and 5. However, the increment is relatively small, an increase 




Fig. 3. ISP efficiency comparison under different peer selection strategies.

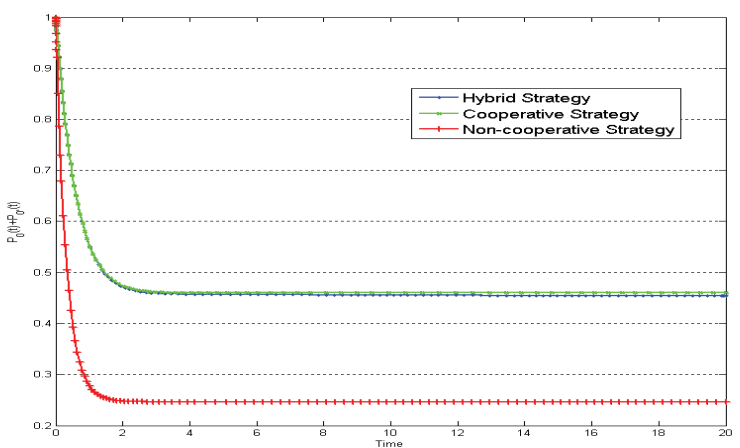

Fig. 5. Cross-ISP traffic reduction ratio comparison under different peer Fig. selection strategies.

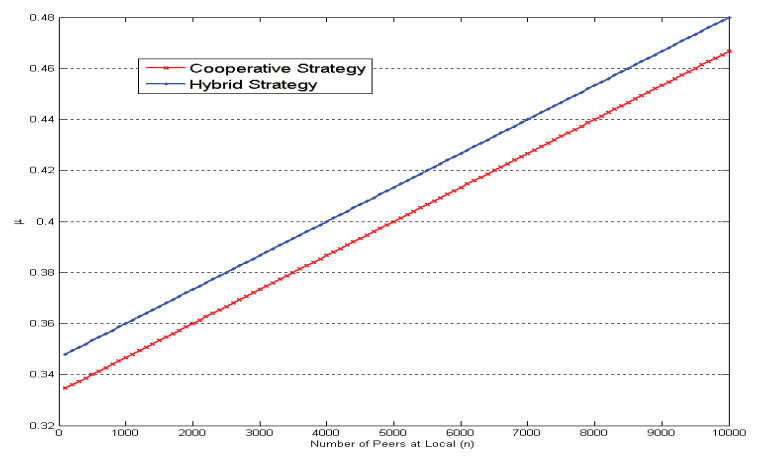

Fig. 7. P2P user rate efficiency comparison under different peer selection Fig. 8. A comparison of benefits of network's and P2P user's with the number strategies.

of $3 \%$ for the value of $\lambda_{r}=0.1$, and the hybrid peering still outperforms the non-cooperative peering strategy greatly. Nevertheless, one of the concerns regarding more costly transit traffic incurred could be raised under the hybrid peering scheme, which can cause revenues loss for some ISPs due to no differentiations between individual domains for the adoption of non-cooperative strategy. As shown later of the economic benefits, desirable benefits of individual ISPs can be maintained if Corollary 1 can be satisfied, however.

Fig. 6 compares the ISP economic benefits under the hybrid and cooperative peering scenario, respectively. As analyzed previously, since the hybrid peering strategy incorporates

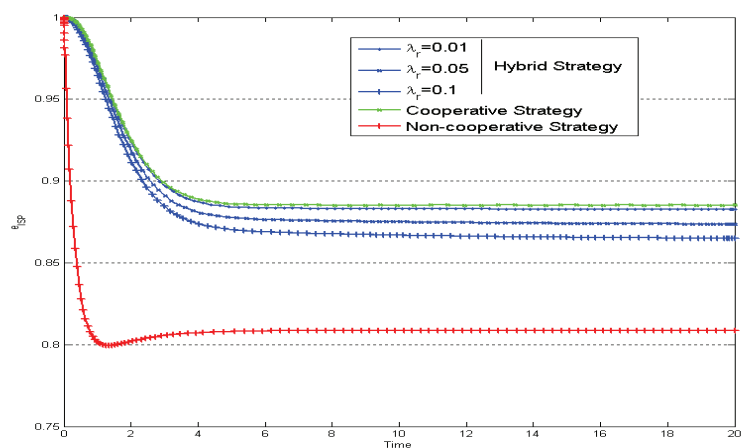

Fig. 4. ISP efficiency comparison with $\lambda_{r}$ varying under different peer selection strategies.

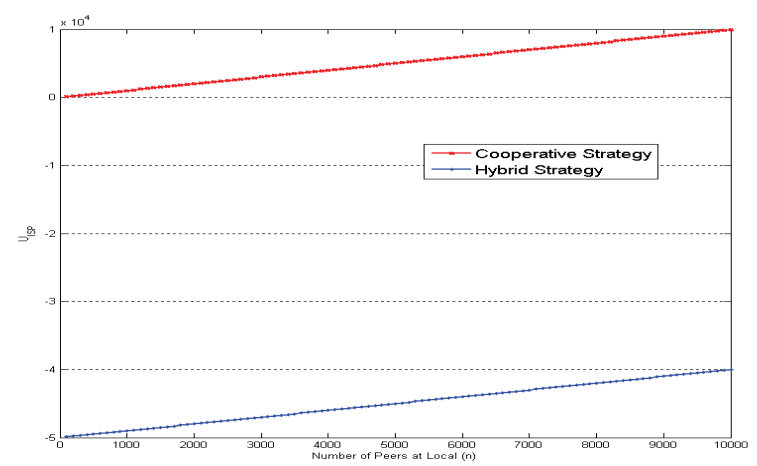

ISP economic benefits comparison under different peer selection strategies.
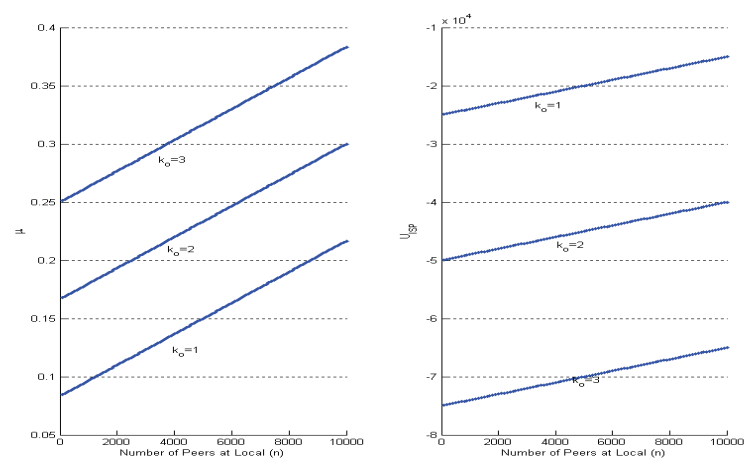
of transit links varying.

random selections of remote domains regarding the same ISPhops into the peering procedure, this may lead to an uncertain increment of the number of transit links involved to carry the P2P traffic. Thus the profits for an ISP can be hardly predicable. The reason is that whether the amount of traffic through its customer ISPs is greater than that through its provider ISPs is unsure, given the stable number of users at local. That is, for some ISPs, e.g., stub ISPs without customer domains subscribing to, the profits can be impacted much more than those of lower tier of ISPs, such as tier-1 ISPs that provide transit connection with customer ISPs for access of the Internet. Therefore, the revenues generated by an ISP 
can experience decrement under hybrid peer selection scenario compared to the all cooperation-based peering scenario, as shown in the figure. However, if specific requirements can be maintained in the hybrid scenario to transfer the $\mathrm{P} 2 \mathrm{P}$ traffic, namely, if Corollary 1 can be met, the profit of an ISP can be guaranteed.

\section{B. P2P User Efficiency Evaluation}

We evaluate the P2P user's efficiency in Fig 7 under different peer selection strategies with $k=4$. The results depicted in the figure demonstrate that users' perceived service quality can be enhanced greatly under hybrid peering strategy, which is consistent with the previous analysis with an increment of $\frac{n_{r} E_{r}[\eta]}{N q} \gamma$. A concern may arise that while localization of P2P traffic can enhance the network efficiency in terms of reduction of costly transit traffic, the service quality perceived by $\mathrm{P} 2 \mathrm{P}$ users could encounter degradation since fewer inter-domain links can be adopted. However, as shown in Fig 8, the varying of the inter-domain links' number can have bigger impact on ISP economic gains, in comparison to the users' rate efficiency. In particular, the increment of inter-domain links can increase the transit traffic cost for the network by $33.4 \%$ for given number of peers at local of around 100. In comparison, the gains for the $\mathrm{P} 2 \mathrm{P}$ users in terms of user efficiency are relatively small, an improvement of only $8.4 \%$ with the increment of the number of inter-ISP links. Thus there may exist concerns that limited number of inter-domain links can impact users' service quality greatly, which cannot be necessarily the case according to our analysis.In comparison, the ISP economic benefits can be enhanced significantly, however. The result further proves the effectiveness of localization promotion from the network side while achieve desirable P2P users' service quality.

\section{CONCLusion}

In this paper, we aim to explore peer selection in a multidomain scenario, with awareness of both cooperative networks and non-cooperative networks coexisting at the same time. A hybrid peer selection scheme is designed for this purpose, incorporating ISP business relationships and other context information, which can achieve better users' perceived service quality, and is able to achieve desirable network efficiency compared to native peer selection strategies. Comprehensive analysis has been performed concerning ISP efficiency, economic benefits and user efficiency in order to systematically analyse different aspects of P2P system behaviours and their implications to the underlying network. In particular, we have derived condition requirements for ISPs to target in order to achieve desirable utilities for both ISPs and P2P systems while reducing cross-ISP traffic. Numerical results show that the hybrid peer selections is able to achieve better performance for P2P users in terms of improved user rate efficiency, while desirable network efficiency can be maintained as the native cooperation-based peering scheme, The proposition in this paper can reduce the risk of congestion probabilities on critical inter-ISP links and also possible failure operations of cooperation strategies, and thus avoid the degradation of $\mathrm{P} 2 \mathrm{P}$ systems performance and maintain the profits of networks.

\section{ACKNOWLEDGMENT}

The research leading to these results has been performed within the UniverSelf project (www.UniverSelf-project.eu) and received funding from the European Community's Seventh Framework Programme (FP7/2007-2013) under grant agreement no 257513.

\section{REFERENCES}

[1] V. Aggarwal, A. Feldmann, and C. Scheideler, Can ISPs and P2P systems co-operate for improved performance?, in Proc. of ACM SIGCOMM Computer Communications Review (CCR), vol. 37, no. 3, pp. 29-40, July 2007.

[2] H. Xie, Y. R. Yang, Y. Liu, A. Krishnamurthy, and A. Silberschatz, $P 4 P$ : Provider portal for applications, in Proc. of ACM SIGCOMM, Aug. 2008.

[3] D. Choffnes and F. Bustamante, Taming the torrent: A practical approach to reducing cross-ISP traffic in P2P systems, in Proc. of ACM SIGCOMM, August 2008.

[4] S. Ren, E. Tan, T. Luo, S. Chen, L. Guo, and X. Z. M. Corporation, TopBT: A topology-aware and infrastructure-independent BitTorrent client, in Proc. of IEEE INFOCOM, Mar. 2010.

[5] J. Seedorf and E. W. Burger, Application-Layer Traffic Optimization (ALTO) Problem Statement, IETF RFC 5693, Oct. 2009.

[6] Z. Dulinski, Cost-driven Peer Rating Algorithm, in Proc. of IEEE International Conference on Communications (ICC), 2011.

[7] Peter Racz, Simon Oechsner and Frank Lehrieder, BGP-Based Locality Promotion for P2P Applications, in Proc. of IEEE 19th International Conference on Computer Communications and Networks (ICCCN), 2010.

[8] Caesar, M., Rexford, J, BGP Routing Policies in ISP Networks, in Proc. of IEEE Network, vol. 19, no. 6, 2005.

[9] S. Ramabhadran and J. Pasquale, Analysis of long-running replicated systems, in Proc. of IEEE INFOCOM '06, Barcelona, Spain, April 2006.

[10] Y. Chawathe, S. Ratnasamy, L. Breslau, N. Lanham, and S. Shenker, Making Gnutella-like P2P systems scalable, in Proc. of ACM SIGCOMM, 2003.

[11] A. Akella, S. Seshan, and A. Shaikh, An Empirical Evaluation of WideArea Internet Bottlenecks, in Proc. of ACM IMC, 2003.

[12] H. Schulze and K. Mochalski, Internet Study 2008/2009, ipoque GmbH Whitepaper, 2009

[13] H. J. Kolbe, O. Kettig, and E. Golic, Monitoring the Impact of P2P Users on a Broadband Operator's Network, in Proc. of IFIP/IEEE IM 2009, Long Island, NY, Jun. 1-5, 2009.

[14] F. Kelly, Charging and Rate Control for Elastic Traffic, European Trans. Telecomm., vol. 8, pp. 33-37, 1997.

[15] T. Basar and R. Srikant, Revenue-Maximizing Pricing and Capacity Expansion in a Many-User Regime, in Proc. of IEEE INFOCOM, pp. 1556-1563, June 2002.

[16] Tobias Hoßfeld, David Hock and Simon Oechsner, Measurement of BitTorrent Swarms and their AS Topologies, Tech. Rep.464, University of Wrzburg, Jan. 2010

[17] J. Erman, A. Mahanti, M. Arlitt, and C. Willianmson, Identifying and discriminating between web and peer-to-peer traffic in the network core, in Proc. of ACM WWW, May 2007.

[18] M. Piatek, H. V. Madhyastha, J. P. John, A. Krishnamurthy, and T. Anderson, Pitfalls for ISP-friendly P2P design, in Proc. of ACM HotNets, Oct. 2009.

[19] R. C. Rumin, N. Laoutaris and X. Yang, Deep Diving into BitTorrent Locality, in Proc. of IEEE INFOCOM, 2011.

[20] John S. Otto, Mario A. Sánchea and David R. Choffnes, On Blind Mice and Elephant, in Proc. of ACM SIGCOMM, 2011.

[21] D. Qiu and R. Srikant, Modeling and performance analysis of bit torrentlike peer-to-peer networks, in Proc. of ACM SIGCOMM, Portland, OR, USA, August 2004.

[22] Rakesh Kumar, Yong Liu, and Keith Ross, Stochastic Fluid Theory for P2P Streaming Systems, in Proc. of IEEE INFOCOM, 2007. 